\title{
Les troubles dépressifs chez les patients atteints de cancer. Une étude marocaine
}

\author{
Depression in cancer patients. A moroccan study
}

\author{
F. Manoudi $\cdot$ R. Chagh $\cdot$ F. Asri $\cdot$ M. Tarwate $\cdot$ I. Tazi $\cdot$ A. Tahiri $\cdot$ N. Bouras
}

Reçu le 20 août 2009 ; accepté le 10 mars 2010

(C) Springer-Verlag France 2010

Résumé La dépression est une affection fréquemment associée à la maladie cancéreuse, sa prévalence varie considérablement du fait de l'hétérogénéité des populations étudiées et des différentes méthodes d'évaluation utilisées. La prévalence de la dépression chez les patients atteints de cancer varie de 0 à $58 \%$. L'étude que nous avons menée avait pour objectifs d'estimer la prévalence de la dépression chez une population de patients atteints de cancer, de décrire leur profil sociodémographique et clinique et de déterminer les facteurs éventuels influençant la survenue de la dépression chez ces patients. L'étude a été menée au niveau du service d'oncologie du CHU Mohamed-VI de Marrakech et a concerné 100 patients ayant des cancers de différents sites et stades. Les instruments utilisés étaient le MINI (DSM-IV) pour poser le diagnostic de la dépression et l'échelle de Beck pour évaluer sa sévérité. Les résultats ont montré que la prévalence de la dépression était de $15 \%$, dont $26,7 \%$ était sévère, $60 \%$ modérée et $13,3 \%$ légère. La dépression était fréquemment rencontrée chez les femmes, d'un âge allant de 37 à 50 ans, mariées, n'ayant jamais été scolarisées ou ayant un niveau universitaire, atteintes d'un cancer de stade localisé, dont la découverte a été faite très précocement (un à trois mois) ou dépassant 49 mois. La dépression a été également plus présente chez les patients qui avaient un entourage familial indifférent à leur maladie, et pour ceux que le coût de la prise en charge ne constituait pas la principale cause de gêne. Une plus grande attention

F. Manoudi $(\bowtie) \cdot$ R. Chagh $\cdot$ F. Asri $\cdot$ M. Tarwate $\cdot$ I. Tazi

Équipe de recherche pour la santé mentale,

faculté de médecine et de pharmacie,

université Caddi-Ayyad,

CHU Mohammed-VI Marrakech, Maroc

e-mail : fatiha.manoudi@gmail.com

A. Tahiri $\cdot$ N. Bouras

Service d'oncologie CHU Mohammed-VI,

Marrakech, Maroc

F. Manoudi

Service de psychiatrie, hôpital Ibn-Nafis,

Marrakech, Maroc devra être prodiguée à ces patients atteints de cancer, avec un dépistage et un traitement précoce de la dépression, afin d'assurer une prise en charge globale et multidisciplinaire et une meilleure qualité de vie. Pour citer cette revue : Psycho-Oncol. 4 (2010).

Mots clés Cancer · Dépression · Prévalence · Sévérité

Abstract Depression is frequently associated with cancer; its prevalence varies considerably because of the heterogeneity of the populations studied and the different methods of assessment used. The prevalence of depression in cancer patients ranges from 0 to $58 \%$. The study that we conducted had objectives to estimate the prevalence of depression in a population of cancer patients, to describe their sociodemographic and clinical profile and to determine the possible factors influencing the occurrence of depression in these patients. The study was conducted at the oncology department of the university hospital Mohammed VI in Marrakech, involving 100 cancer patients assigns different sites and stadiums. The instruments used were MINI (DSM-IV) for the diagnosis of depression, and Beck Depression Inventory to assess its severity. The results showed that the prevalence of depression was $15 \%$, in which $26,7 \%$ was severe, $60 \%$ moderate and $13,3 \%$ mild. The depression was frequently encountered among women, from 37 to 50 years old, married, had never been enrolled in school or with a university degree, with a localized cancer, whose discovery was made very early (1 to 3 months) or which exceeds 49 months. The depression was also frequently present in patients who had an indifferent familial entourage to their disease, and for those that the cost of care was not the main cause of discomfort. Greater attention should be given to these cancer patients, with screening and early treatment of depression, for overall and multidisciplinary care and better quality of life. To cite this journal: Psycho-Oncol. 4 (2010).

Keywords Cancer $\cdot$ Depression $\cdot$ Prevalence $\cdot$ Severity 


\section{Abréviations \\ HADS Hospital Anxiety and Depression Scale \\ EORTC European Organisation for Research and Treatment of Cancer \\ SCID Structured Clinical Interview for DSM III-R \\ Endicott the Interview criteria for major depression modified by Endicott for cancer patients (Endicott 1984) \\ HAMD Hamilton Depression Rating Scale}

\section{Introduction}

La découverte d'un cancer marque souvent l'entrée dans un lourd et long processus de soins. Si le corps doit affronter de rudes épreuves, la psyché n'est pas épargnée.

La dépression chez les patients cancéreux est souvent non reconnue, négligée et sous-diagnostiquée ; d'une part parce que des sentiments de tristesse et de découragement sont souvent considérés comme normaux et adaptés face à la prise de conscience d'un diagnostic aussi lourd de conséquences que le cancer, et d'autre part parce que de nombreux signes sont communs à la maladie cancéreuse et à la dépression comme la perte de poids, la fatigue ou les troubles du sommeil [26].

L'objectif de notre travail est de déterminer la prévalence de la dépression au sein d'une population de patients atteints de cancer pris en charge au niveau du service d'oncologie au CHU Mohamed-VI de Marrakech au Maroc, ainsi que de déterminer les éventuels facteurs influençant la survenue de la dépression chez ces patients.

\section{Matériel et méthodes}

Nous avons mené une étude transversale à visée descriptive ayant concerné un échantillon de 100 patients, tous atteints de cancers, de localisations différentes, vus en consultations médicales ou en hôpital de jour au service d'oncologie pour des cures thérapeutiques.

Un questionnaire a été élaboré par le service de psychiatrie (CHU Mohamed-VI). Les questions ont été posées en langue arabe dialectale.

Pour l'étude de la dépression, nous avons utilisé deux types d'instruments. L'entretien structuré qui reprend les critères du DSM-IV (le Mini International Neuropsychiatric Interview) pour poser le diagnostic de la dépression, et l'échelle de Beck, pour évaluer la sévérité de la dépression.

L'enquête a eu lieu entre juillet et décembre 2007. Elle a porté sur 112 patients. Cinq patients avaient refusé de participer à l'étude, trois patients avaient un état général très altéré et quatre patients avaient un problème de langage (parlant berbère). Le recueil des données a été effectué avec le respect de l'anonymat des patients et de la confidentialité de leurs informations.

Si le diagnostic d'état dépressif majeur était porté lors de l'évaluation, une prise en charge spécialisée était proposée.

L'analyse statistique a été faite grâce au logiciel ÉpiInfo ${ }^{\text {TM }}$ $6.04 \mathrm{~d} \mathrm{Fr}$., le seuil de signification a été fixé à $5 \%$.

\section{Résultats}

La majorité des patients était de sexe féminin (65\%). L'âge moyen était de 45,32 ans, avec des extrêmes allant de 15 à 72 ans, la tranche d'âge entre 37 et 50 ans était la plus représentée $(43 \%)$. La majorité des patients étaient mariés (72\%). Presque les deux tiers n'étaient jamais scolarisés (62\%), et $6 \%$ des patients seulement avaient un niveau universitaire. La moitié des patients de notre échantillon était des femmes au foyer, plus de la moitié avaient un revenu mensuel familial inférieur à 200 euros $(54 \%)$ avec aucune couverture sociale dans $77 \%$ des cas. La majorité des patients avaient des enfants âgés de 5 à 15 ans (33\%), $28 \%$ avaient des enfants dont l'âge est supérieur à 15 ans, et $19 \%$ des patients avaient des enfants âgés de moins de cinq ans. Deux patients avaient un antécédent de cancer.

Le premier avait un lymphome non hodgkinien, diagnostiqué il y a 36 mois, traité par chimiothérapie, ayant bien évolué. Actuellement, il a un lymphome hodgkinien. Le deuxième avait probablement un cancer ostéochondral de la jambe droite (site et type non précisés par le patient), traité par amputation de la jambe, il y a 36 mois, ayant bien évolué aussi. Actuellement, il a un cancer du larynx.

Trois patients avaient un antécédent de cancer familial similaire. La localisation majoritaire retrouvée était la localisation mammaire (31\%), suivie de la localisation au niveau du tube digestif (21\%) et $13 \%$ des cancers étaient localisés au niveau de l'appareil génital féminin. Les tumeurs hématologiques représentaient $10 \%$ de l'ensemble des localisations.

Les 13 autres localisations étaient représentées par cinq localisations osseuses, deux au niveau des fosses nasales, un cancer du larynx, un sarcome de la nuque, un méningiome, un cancer du testicule et un foyer primitif inconnu.

La grande majorité des patients avaient une tumeur solide (90\%), avec un cancer de stade localement avancé dans $49 \%$ des cas et $34 \%$ avaient un cancer au stade métastatique. Pour les tumeurs hématologiques, sept patients sur dix avaient un stade diffus. Le délai moyen de découverte du cancer était de 14,74 mois avec des extrêmes allant d'un à 120 mois.

Il n'y avait pas de changement d'attitude de l'entourage chez la majorité des patients ( $67 \%$ ), et $33 \%$ rapportaient un changement; il s'agissait soit d'un changement positif (26\%) 
sous forme de soutien social, familial, moral et financier, soit un changement négatif ( $7 \%$ ) avec désintérêt, éloignement par crainte de contagiosité, soit dans $67 \%$ des cas une attitude indifférente. Concernant la pratique religieuse ; chez $77 \%$ des patients il n'y avait aucun changement dans leur pratique religieuse (prières, lecture de Coran...), $18 \%$ avaient augmenté leur pratique et $7 \%$ avaient rapporté une diminution, voire un arrêt des prières. La gêne des patients interrogés était due principalement à trois éléments ; le coût de la prise en charge (58 \%), les symptômes physiques (55\%) et les effets secondaires de la chimiothérapie (45\%). Presque les deux tiers des patients recevaient un traitement curatif (62\%), notamment un traitement curatif systémique chimiothérapie seule ou associée à un traitement locorégional (chirurgie ou radiothérapie ou les deux) [55\%], alors que $8 \%$ seulement recevaient un traitement locorégional seul. Le délai moyen du début du traitement était de 12,83 mois avec un minimum d'un mois et un maximum de 120 mois. Les patients qui étaient en stabilisation et ceux qui étaient en rémission étaient presque à nombre égal ( 34 et $38 \%$ respectivement), $21 \%$ des patients étaient en rechute et $7 \%$ venaient juste de commencer leur traitement (moins d'une semaine). Plus de la moitié des patients savaient qu'ils avaient un cancer (54\%), et la majorité (78\%) croyait que leur maladie est curable, contre $9 \%$ qui la croyaient incurable, alors que $13 \%$ ne pouvaient donner aucune estimation. Selon le MINI (DSM-IV), $15 \%$ des malades présentaient un épisode dépressif majeur (EDM) au moment de l'enquête, et seulement $6 \%$ avaient présenté un EDM dans leur passé.

Selon l'échelle de Beck, 26,7 \% des patients déprimés avaient une dépression sévère, $60 \%$ avaient une dépression modérée et 13,3\% avaient une dépression légère (Fig. 1).

L'EDM est retrouvé un peu plus chez les femmes $(15,4 \%)$ que chez les hommes $(14,3 \%)$. Cette différence est statistiquement significative $(p=0,015)$. Le quart

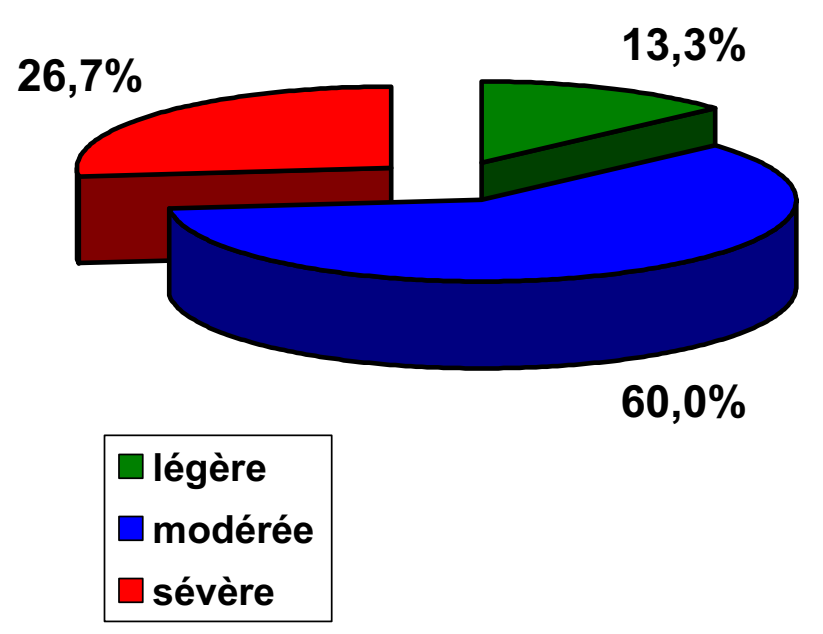

Fig. 1 Sévérité de la dépression des patients âgés de 37 à 50 ans ont un EDM et 4,2\% des patients âgés de moins de 36 ans ont un EDM. Cela est statistiquement significatif.

L'analyse bivariée a mis en évidence certaines corrélations positives $(p<0,005)$ :

- les patients mariés présentaient plus de dépression $(19,4 \%)$. Alors que chez les sujets célibataires et veufs on n'a pas retrouvé d'EDM ;

- la prévalence de dépression retrouvée est égale pour les patients n'ayant aucun niveau d'instruction et pour ceux ayant un niveau universitaire $(16,1$ et $16,7 \%)$, alors qu'elle est de $12,5 \%$ chez les patients ayant un niveau primaire et secondaire ;

- $15 \%$ des patients ayant une comorbidité (autre maladie chronique) étaient déprimés, et $33 \%$ de ceux ayant un antécédent médical étaient déprimés ;

- les patients qui étaient déprimés parmi ceux qui sont gênés par le coût de la prise en charge représentaient $13,8 \%$ et parmi ceux que les frais de la prise en charge ne gênaient pas représentaient $17,9 \%$. Cette différence est à la limite de la signification.

Aucune corrélation significative n'a été retrouvée avec les autres variables, néanmoins l'EDM était plus noté :

- chez les femmes $(15,4 \%)$ que chez les hommes $(14,3 \%)$;

- chez les patients qui ont des enfants dont le plus jeune est âgé de moins de cinq ans $(26,3 \%)$;

- chez les femmes au foyer (10\%), contre $4 \%$ chez les travailleurs actifs. On n'a pas trouvé d'EDM chez les étudiants et les sujets sans profession;

- les patients dont le revenu mensuel familial est entre 500 et 1000 euros étaient plus déprimés $(40 \%)$;

- la prévalence de l'EDM dans les cancers solides était de $16,7 \%$, par contre aucun cas de dépression n'était retrouvé chez les patients ayant un cancer hématologique. C'est dans le stade localisé qu'on a retrouvé plus de dépression : 28,6\%. La prévalence de la dépression dans le stade localement avancé est de $14,3 \%$, et dans le stade métastatique de $17,6 \%$;

- les patients ayant une attitude négative de l'entourage avaient plus de dépression (14,3\%);

- les patients ayant augmenté leur pratique religieuse après la découverte du cancer avaient plus de dépression $(27,8 \%)$;

- aucun des patients gênés par sa perte d'autonomie n'était déprimé ;

- les patients gênés par les effets secondaires du traitement qui étaient déprimés représentaient $22,2 \%$, contre $9,6 \%$ de dépression chez les patients qui n'étaient pas gênés. L'EDM est plus retrouvé chez les patients qui rapportaient une gêne par les vomissements comme effet secondaire du traitement $(23,1 \%)$. Un quart des patients 
qui sont gênés par l'asthénie avaient un EDM, et seulement $7,1 \%$ des patients qui ne rapportaient pas de gêne étaient déprimés ;

- l'EDM était présent de façon égale chez les patients recevant un traitement palliatif ou curatif ( 8 et $7 \%$ respectivement), et de façon presque égale aussi quel que soit le délai de début de traitement. Par ailleurs, chez les patients qui l'avaient démarré il y a 49 mois et plus, on n'a pas trouvé de dépression;

- on a retrouvé le moins de dépression chez les patients qui croyaient que leur maladie est curable (12,8 \%), alors que chez ceux qui croyaient que leur maladie est incurable et ceux qui ne pouvaient rien dire, la prévalence est presque égale (22,2 et $23,1 \%$ respectivement) ;

- on a aussi noté plus de dépression chez les patients qui savaient qu'ils avaient un cancer (12\%), et chez seulement $3 \%$ de ceux qui l'ignoraient ;

- aucun des patients ayant un antécédent d'un autre cancer n'était déprimé ;

- c'est dans le stade localisé qu'on a retrouvé plus de dépression $(28,6 \%)$; la prévalence de la dépression dans le stade localement avancé est de $14,3 \%$, et dans le stade métastatique de $17,6 \%$;

- concernant le délai de découverte de cancer, les patients qui avaient le plus de dépression étaient ceux dont le diagnostic a été posé il y a plus de 49 mois $(66,7 \%)$ (Fig. 2) ;

- les patients ayant un cancer du poumon et ceux ayant un cancer du sein avaient le taux le plus élevé de dépression avec respectivement 57,1 et $19,4 \%$ (Fig. 3) ;

- le délai moyen de début du traitement chez les patients déprimés était de 9,21 mois avec un écart-type de 10,17 ; un minimum d'un mois et un maximum de 32 mois. Chez les patients non déprimés, cette moyenne était plus élevée (13,47 mois), avec un écart-type de 18,93, un minimum d'un mois et un maximum de 120 mois ;
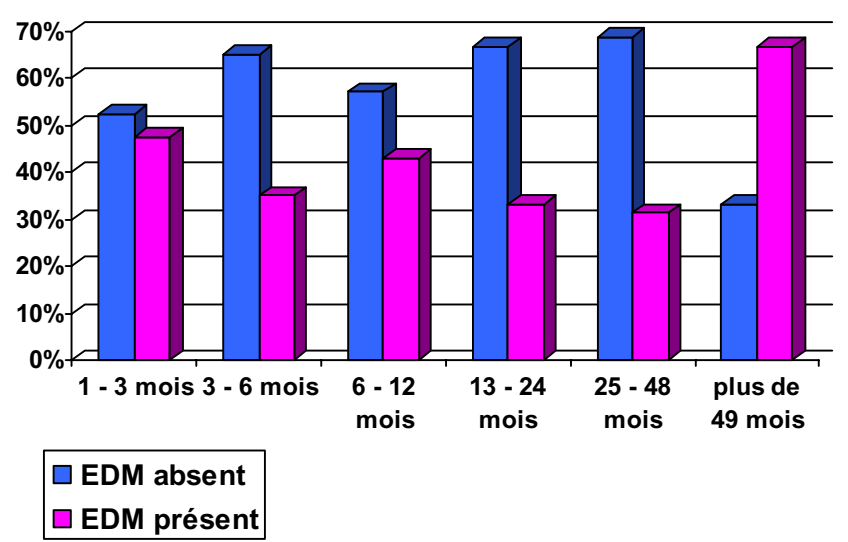

Fig. 2 Prévalence de la dépression selon le délai de découverte du cancer

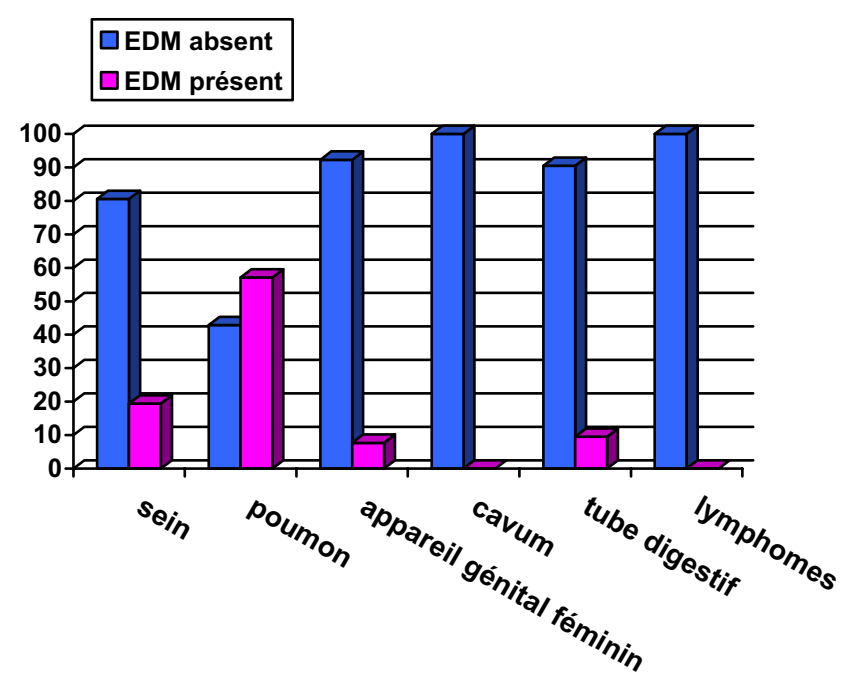

Fig. 3 Prévalence de la dépression selon le site du cancer

- les patients les plus déprimés étaient en stabilisation $(26,5 \%)$, et les moins déprimés étaient en rémission $(7,9 \%)$.

\section{Discussion}

La dépression est la réaction psychiatrique la plus fréquente chez les patients atteints de cancer [19]. Chez ces patients, le diagnostic de dépression présente souvent des difficultés évidentes du fait des chevauchements qui existent entre les symptômes somatiques dus à la maladie cancéreuse et aux effets secondaires des traitements, avec ceux de la dépression.

En 2002, le nombre de nouveaux cas de cancer dans le monde est estimé à 10,9 millions, avec 6,7 millions de décès [12].

En France, c'est la deuxième cause de mortalité (23\%) avec 120000 décès/an [17].

$\mathrm{Au}$ Maroc, le premier registre des cancers de la région du grand Casablanca a estimé 30500 nouveaux cas chaque année, ce qui correspond à une incidence standardisée globale de 101,7 nouveaux cas/100 000 habitants par an [4].

La prévalence de la dépression dans notre étude est de $15 \%$, ce résultat se rapproche de celui trouvé dans d'autres études similaires (Tableau 1).

L'influence du sexe a fait l'objet de plusieurs études. Plusieurs auteurs n'ont pas trouvé de différence significative $[2,10,14]$. La plus grande prévalence de dépression retrouvée dans notre étude chez les femmes pourrait être expliquée par le fait que dans la population générale, la prévalence des troubles dépressifs est deux fois supérieure chez le sexe féminin. 


\begin{tabular}{|c|c|c|c|c|}
\hline Auteur & Année & Population étudiée & Méthode utilisée & $\begin{array}{l}\text { Prévalence } \\
\text { de la dépression }\end{array}$ \\
\hline Alexander et al. (Inde) [3] & 1993 & $\begin{array}{l}\text { Sites et stades variés } \\
\quad n=60\end{array}$ & $\begin{array}{l}\text { DSM III-R, } \\
\text { clinical interview }\end{array}$ & $13 \%$ \\
\hline Aass et al. (Norvège) [1] & 1997 & $\begin{array}{l}\text { Cancers de sites variés } \\
\quad n=716\end{array}$ & HADS, EORTC & $9 \%$ \\
\hline Chochinov et al. [9] (Canada) & 1997 & $\begin{array}{l}\text { Stade terminal } \\
\quad n=197\end{array}$ & $\begin{array}{l}\text { BECK, semi-structured } \\
\text { interview }\end{array}$ & $\begin{array}{l}12,2 \% \\
7,6 \% \text { majeure } \\
4,6 \% \text { mineure }\end{array}$ \\
\hline Skarstein et al. (Norvège) [25] & 2000 & $\begin{array}{l}\text { Sites variés de cancer } \\
\quad n=568\end{array}$ & HADS, EORTC & $9 \%$ \\
\hline Chen et Chang (Taïwan) [6] & 2000 & $\begin{array}{l}\text { Tumeurs solides et liquides } \\
\quad n=203\end{array}$ & HADS & $20 \%$ \\
\hline Pascoe et al. (Australie) [21] & 2000 & $\begin{array}{l}\text { Sites variés ambulatoires } \\
\quad n=504\end{array}$ & HADS & $7,1 \%$ \\
\hline Breitbart et al. [5] & 2000 & $\begin{array}{l}\text { Sites variés en hospitalisation } \\
\quad n=92\end{array}$ & DSM-III & $16 \%$ \\
\hline Ciaramella et Poli (Italie) [10] & 2001 & $\begin{array}{l}\text { Sites variés de cancers } \\
\quad n=100\end{array}$ & $\begin{array}{l}\text { Interview, SCID, } \\
\text { Endicott, HAMD }\end{array}$ & $\begin{array}{l}49 \% \mathrm{SCID} / 29 \% \\
\text { Endicott } / 28 \% \\
\text { les deux }\end{array}$ \\
\hline Akechi et al. (Japan) [2] & 2004 & $\begin{array}{l}\text { Stade terminal } \\
\quad n=209\end{array}$ & $\begin{array}{l}\text { Structured clinical interview } \\
\text { DSM IV, HADS }\end{array}$ & $6,7 \%$ \\
\hline Wilson et al. (Canada) [31] & 2007 & $\begin{array}{l}\text { Cancers en soins palliatifs } \\
\quad n=381\end{array}$ & Semi-structured Interview & $13,1 \%$ \\
\hline $\begin{array}{l}\text { Krähenbühl et al. } \\
\text { (Allemagne) [16] }\end{array}$ & 2007 & $\begin{array}{l}\text { Cancers de différents types et } \\
\text { stades ambulatoires } \\
n=109\end{array}$ & Distress thermometer & $20,2 \%$ \\
\hline Notre étude (Maroc) & 2007 & $\begin{array}{l}\text { Différents sites et stades } \\
\qquad n=100\end{array}$ & SCID, BECK & $\begin{array}{l}15 \% \\
26,7 \% \text { sévère, } \\
60 \% \text { modérée, } \\
13,3 \% \text { légère }\end{array}$ \\
\hline
\end{tabular}

Nous avons trouvé dans notre étude que la prévalence la plus élevée de dépression est celle retrouvée chez les sujets âgés de 37 à 50 ans (25,6\%). Akechi et al. [2], et Wilson et al. [31] rapportent que le jeune âge est un facteur significativement associé à la dépression. Cela peut être expliqué par la survenue d'un traumatisme émotionnel violent chez les plus jeunes, alors que les plus âgés considèrent cette maladie comme grave mais l'intègrent volontiers dans le processus de vieillissement parvenant ainsi plus facilement que les jeunes à une conciliation avec leur maladie.

La dépression est plus retrouvée chez nos patients mariés $(19,4 \%)$. Contrairement à nos résultats, Bardwell et al. [30] ont trouvé sur une population de patientes atteintes de cancer du sein que les femmes mariées étaient moins déprimées que les célibataires.

Nos patients ayant un niveau universitaire, ainsi que ceux n'ayant jamais été scolarisés étaient statistiquement les plus déprimés (16,7 et 16,1\% respectivement). Pour Akechi et al. [2], une longue instruction (supérieure ou égale à neuf ans) est un facteur signifiant associé à la dépression. Kai-hoi Sze et al. [14], Bardwell et al. [30], Ell et al. [11] et Ciaramella et Poli [10] n'ont pas trouvé de corrélation significative de la dépression avec le niveau d'instruction.

Nous avons trouvé que l'existence de comorbidité est significativement liée à la dépression. Yun et al. [32], en 2007 a démontré dans son étude que les comorbidités augmentent le risque de dépression. Ell et al. [11], en 2005 précisent que l'existence de comorbidité articulaire est significativement liée à la dépression.

Concernant la localisation du cancer ; il existe des localisations très associées à la dépression (cancer du poumon et du sein dans notre étude). Massie [18], d'après une synthèse d'un ensemble d'études, rapporte que les cancers très associés à la dépression sont les cancers 
oropharyngés avec une prévalence de dépression allant de 22 à $57 \%$, pancréatiques (33 à $50 \%$ ), mammaires $(1,5$ à $46 \%)$ et pulmonaires (11 à $44 \%)$. Une moindre prévalence de la dépression est retrouvée dans les cancers du côlon (13 à $25 \%$ ), gynécologiques (12 à $23 \%$ ) et les lymphomes ( 8 à $19 \%$ ). Plusieurs hypothèses peuvent expliquer ces constatations :

- la première est simple : les cancers du poumon et du pancréas sont connus pour avoir un sombre pronostic, les malades qui en sont atteints peuvent le savoir et souffrir plus ;

- la seconde hypothèse est qu'il s'agit d'une forme de syndrome paranéoplasique. La présence en grande quantité de neuropeptides au niveau du tractus gastro-intestinal et au niveau du cerveau suggère des interactions par lesquelles la tumeur produirait une substance capable d'altérer les amines biogènes cérébrales qui contrôlent l'humeur $[8,13]$;

- la troisième hypothèse est que la dépression résulterait d'une interférence immunologique avec l'activité sérotoninergique. Un anticorps induit contre une protéine relâchée par les cellules cancéreuses pourrait, étant donné l'activité croisée avec le système nerveux central, se fixer sur les récepteurs sérotoninergiques et les bloquer, favorisant ainsi l'apparition d'une dépression.

Les stades successifs de la maladie vont conduire d'abord à l'apparition, puis à l'entretien du syndrome dépressif. C'est ainsi que l'impossibilité de poursuivre une activité quotidienne normale et la déchéance physique liée à la progression de la maladie peuvent entraîner une dépression. En effet, Ciaramella et Poli [10] ont rapporté dans leur étude une augmentation significative de la dépression chez les patients au stade de métastases. Aass et al. [1] ont retrouvé une corrélation significative du stade avec la dépression, $14 \%$ des patients ayant des métastases à distance étaient déprimés, contre $6 \%$ chez ceux qui étaient en stade localisé ou locorégional. Dans notre étude, ce sont les patients en stade localisé qui ont significativement le plus de dépression (28,6\%) et seulement $17,6 \%$ des patients au stade métastatique ; cela pourrait être expliqué par le fait que les patients ayant des métastases étaient dans les deux tiers des cas en rechute, ce qui signifie qu'ils avaient assez de temps pour s'adapter psychologiquement avec leur maladie cancéreuse et l'éventualité d'une progression, contrairement à ceux qui étaient en stade localisé. Concernant la corrélation entre le délai moyen de découverte du cancer et la dépression, plusieurs études ne trouvent pas de différence significative avec une population-témoin au-delà de deux ans [15]. En phase des premiers symptômes, le malade est confronté à une nouvelle réalité inattendue, douloureuse et choquante quelquefois. Les premiers symptômes ne coïncident pas nécessairement dans le temps avec une décision de consulter.
Le déni, mécanisme par lequel un état de connaissance douloureux ignoré, banalisé ou évacué, peut être suspecté en cas de retard de diagnostic [29]. Plusieurs facteurs, autres que le déni, sont invoqués pour expliquer ce délai comme une moindre conscience de ce qu'est le cancer et de ses implications ou une peur consciente du cancer, allant de celle d'être examiné à celle de souffrir ou d'être mutilé. Les préoccupations liées au cancer concernent principalement les conséquences relatives aux répercussions familiales, la mort et la douleur.

Parmi les symptômes physiques, la douleur est fréquemment associée à la maladie cancéreuse, il est souvent lié à la progression de la maladie. Plusieurs auteurs ont évalué ce paramètre. Wilson et al. [31] ont révélé que 49,5\% des patients qui se plaignent de douleur sont déprimés. Cela est statistiquement significatif. Pour Akechi et al. [2], Chen et Chang [7], Ciaramella et Poli [10] et Ell et al. [11], la présence de douleur est corrélée significativement avec la dépression. Les patients déprimés avaient plus de douleur que les non déprimés.

Il apparaît que la qualité de l'entourage est un facteur crucial dans l'apparition de la dépression. En effet, plus le malade aura du mal à communiquer ses angoisses, plus il se sentira isolé, et plus il sera sensible à la dépression [24,28].

Plusieurs études ont évalué le rôle de la religion dans l'exagération ou l'atténuation des troubles dépressifs chez les patients cancéreux, mais ce paramètre fut étudié de différentes manières, rendant difficile sa comparaison d'une étude à une autre. Wilson et al. [31], ont trouvé moins de dépression chez les patients ayant une religion organisationnelle, par rapport à la religion non organisationnelle et subjective. Thuné-Boyle et al. [27] ont fait une synthèse de 18 études évaluant l'impact de la religion, ils ont trouvé que sept études d'entre elles rapportaient un effet bénéfique, quatre études rapportaient un effet nuisible, et les sept autres n'avaient trouvé aucune corrélation significative.

Concernant la connaissance et le pronostic de la maladie cancéreuse ; Alexander et al. [3], en 2003, ont trouvé que les patients ignorant le diagnostic représentaient $33 \%$, et qu'ils étaient significativement moins déprimés que ceux qui étaient au courant du diagnostic de cancer. Dans cette même étude, Alexander et al. rapportent que $82 \%$ des patients considéraient que leur maladie est curable ; ces patients optimistes avaient considérablement moins de dépression que les autres. Bardwell et al. [30], Pinquart [22] et al., et Mystakidou et al. [20] ont mis en évidence que les sujets les moins optimistes étaient les plus déprimés.

\section{Conclusion}

La maladie cancéreuse est en effet à l'origine d'une série de réactions qui peuvent être cognitives, émotionnelles et 
comportementales ; ces réactions diffèrent d'une phase à une autre, durant l'évolution de la maladie cancéreuse. L'adaptation à la maladie cancéreuse est aussi, bien sûr, très variable d'un individu à un autre [23].

À la phase du diagnostic, la confirmation du cancer est souvent associée dans l'esprit du patient à une sentence de mort. Les préoccupations sont d'abord existentielles, pensées relatives à la mort, sentiment de vulnérabilité, détresse émotionnelle (pessimisme, désespoir, anxiété) s'associent à une remise en question des relations avec le monde environnant, qu'il soit familial, social ou professionnel. Le diagnostic ouvre une période de crise individuelle et interpersonnelle. La peur, l'anxiété et la dépression sont les sentiments les plus fréquemment déclenchés par la maladie, les sentiments d'impuissance et de solitude s'y associent parfois pour aboutir à un désespoir et à un pessimisme pas toujours justifiés par la situation clinique. La préservation d'un niveau d'estime de soi suffisant permet à beaucoup de patients de poursuivre leur vie, de collaborer activement au traitement et à certains de réagir comme s'ils relevaient un défi. En phase de diagnostic et tout au long de la maladie, le thème de la mort est présent mais souvent masqué dans le discours des patients.

En phase du traitement, la détresse des malades est fréquemment liée au caractère invasif des méthodes d'investigations et de traitement.

Nous avons, grâce à cette étude, pris conscience du degré de détresse psychologique de ces patients, et de l'importance primordiale de dépister des troubles dépressifs et de les traiter précocement, dans le cadre d'une prise en charge globale et multidisciplinaire pour une meilleure qualité de vie du patient.

Conflit d'intérêt : les auteurs déclarent ne pas avoir de conflit d'intérêt.

\section{Références}

1. Aass N, Fossa SD, Dahl AA, Moe TJ (1997) Prevalence of Anxiety and Depression in Cancer Patients Seen at the Norwegian Radium Hospital. Eur J Cancer 33(10):1597-604

2. Akechi T, Okuyama T, Sugawara Y, et al (2004) Major depression, adjustment disorders, and post-traumatic stress disorder in terminally ill cancer patients: associated and predictive factors. J Clin Oncol 22:10

3. Alexander PJ, Dinesh N, Vidyasagar MS (1993) Psychiatric morbidity among cancer patients and its relationship with awareness of illness and expectations about treatment outcome. Acta Oncol 32:623-6

4. Benider A (2004) Résultats du premier registre des cancers de la région du grand Casablanca

5. Breitbart W, Rosenbeld B, Pessin H, et al (2000) Depression, hoplessness, and desire for hastened death in terminally ill patients with cancer. JAMA 284:2907-11
6. Chen ML, Chang HK (2004) Physical symptom profiles of depressed and non depressed patients with cancer. Pall Med 18(8):712-8

7. Chen M, Chang H, Yeh C (2000) Anxiety and depression in Taiwanese cancer patients with and without pain. J Adv Nurs 32:944-51

8. Chochinov HM (2001) Depression in cancer patients. Lancet Oncol 2:499-505

9. Chochinov HM, Wison KG, Enns M, Lander S (1997) "Are you depressed?" Screening for depression in the terminally ill. Am J Psychiatry 154:674-6

10. Ciaramella A, Poli P (2001) Assessement of depression among cancer patients: the role of pain, cancer type and treatment. P O 10:156-65

11. Ell K, Sanchez K, Vourlekis B, et al (2005) Depression, correlates of depression, and receipt of depression care among low-income women with breast or gynecologic cancer. J Clin Oncol 23(13):3052-60

12. Hakulinen T, Hakama M, Sankila R, et al (2002) Cancer incidence in five continents, vol. VIII. Eds. IARC Scientific Publications $\mathrm{n}^{\mathrm{o}}$ 155. International Agency for Research on Cancer, Lyon, pp. 328-9

13. Irwin MR (2007) Depression and risk of cancer progression: an elusive link. J Clin Oncol. Jun 10;25(17):2343-4

14. Kai-hoi Sze F, Wong E, Woo J, Lo R (2000) Do pain and disability differ in depressed cancer patients? Palliative Medicine 14(1):11-7

15. Kendal WS (2007) Suicide and cancer: a gender-comparative study. Ann Oncology 18(2):381-7

16. Krähenbühl A, Zwahlen D, Knuth A, et al (2007) Prevalence of anxiety and depression in cancer outpatients and their spouses. Schweiz Rundsch Med Prax 96(24):973-9

17. Lévy V, Lévy-Soussan M (1996) Cancérologie. Éditions Estem et Med-Line; 275 p. Collection Med-Line. ISBN 2-909455-17-3

18. Massie MJ (2004) Prevalence of depression in patients with cancer. Journal of the National Cancer Institute; monographs 32

19. Massie MJ, Gagnon P, Holland JC (1994) Depression and suicide in patients with cancer. J Pain Symptom Manage 9(5): 325-40

20. Mystakidou K, Tsilika E, Parpa E, et al (2007) Exploring the relationships between depression, hopelessness, cognitive status pain and spirituality in patients with advanced cancer. Arch Psychiatr Nurs 21(3):150-61

21. Pascoe S, Edelman S, Kidman A (2000) Prevalence of psychological distress and use of support services by cancer patients at Sydney hospitals. Aust N Z J Psychiatry 34:785-91

22. Pinquart M, Fröhlich C, Silbereisen RK (2007) Optimism, pessimism, and change of psychological well-being in cancer patients. Psychol Health Med 12(04):421-32

23. Razavi D, Delvaux N (1998) Psycho-oncologie: le cancer, le malade et sa famille. $2^{\mathrm{e}}$ édition, Masson, pp 66-92

24. Schroevers MJ, Ranchor AV, Sanderman R (2003) The role of social support and self-esteem in the presence and course of depressive symptoms: a comparison of cancer patients and individuals from the general population. Social Science and Medicine 57:375-85

25. Skarstein J, Aass N, Fossa SD, et al (2000) Anxiety and depression scale and European Organization for Research and Treatment of Cancer Core Quality of Life Questionnaire. J Psychosom Res 49:27-34

26. Spiegel D, Giese-Davis J (2003) Depression and cancer: Mechanisms and disease progression. Biol Psychiatry 54:269-82

27. Thuné-Boyle IC, Stygall JA, Keshtgar MR, Newman SP (2006) Do religious/spiritual coping strategies affect illness adjustment 
in patients with cancer? A systematic review of the literature. Social Science and Medicine 63:151-64

28. Tuinman MA, Hoekstra HJ, Fleer J, et al (2006) Self-esteem, social support, and mental health in survivors of testicular cancer: A comparison based on relationship status. Urologic Oncology 24:279-86

29. Vos MS, Haes JCJM (2006) Denial in cancer patients, an explorative review. PO. DOI: 10.1002/pon.1051

30. Bardwell WA, Natarajan L, Dimsdale JE, et al (2006) Objective cancer-related variables are not associated with depressive symptoms in women treated for early-stage breast cancer. J Clin Oncol 24(16):2420-7

31. Wilson KG, Chochinov HM, Skirko MG, et al (2007) Depression and anxiety disorders in palliative cancer care. J Pain Symptom Manage 33(2):118-29

32. Yun YH, Kim SH, Lee KM, et al (2007) Age, sex, and comorbidities were considered in comparing reference data for healthrelated quality of life in the general and cancer populations. J C Epidemiology 60:1164-75 